\title{
A Performance Comparison of Interference Alignment and Opportunistic Transmission with Channel Estimation Errors
}

\author{
Min Ni, D. Richard Brown III \\ Department of Electrical and Computer Engineering \\ Worcester Polytechnique Institute \\ 100 Institute Rd, Worcester, MA 01609 \\ Email: $\{$ minni, drb $\} @$ wpi.edu
}

\begin{abstract}
This paper compares interference alignment and opportunistic transmission for a 3-user single-input single-output (SISO) interference channel in terms of average sum rate in the presence of channel estimation errors. In the case of interference alignment, channel estimation errors cause interference leakage which consequently results in a loss of achievable rate. In the case of opportunistic transmission, channel estimation errors result in a non-zero probability of incorrectly choosing the node with the best channel. The effect of these impairments is quantified in terms of the achievable average sum rate of these transmission techniques. Analysis and numerical examples for independent and identically distributed fading channels show that SISO interference alignment can achieve better average sum rate with good channel estimates and at high SNR whereas opportunistic transmission provides better performance at low SNR and/or when the channel estimates are poor.
\end{abstract}

Index Terms-Interference alignment, opportunistic transmission, channel estimation errors

\section{INTRODUCTION}

Wireless networks are interference-limited due to the increasing number of users that need to share the spectrum to achieve the high-rate communication. The problem of achieving efficient communication in an interference channel has attracted much research activity in recent years. The growing demands on wireless networks, for example, $4 \mathrm{G}$ networks including WiMAX and 3GPP Long Term Evolution (LTE), to support high data rates and high capacity has driven the need to develop efficient interference management techniques [1], [2].

Conventional interference management approaches such as interference avoidance divide the channel resources among the transmitters, e.g. using time division such that only one node transmits at a time [3]. When the receive nodes can measure and feedback the channel quality, the transmit nodes can transmit opportunistically by using the best available channel at each instant in time [4]. An advantage of opportunistic transmission is that this technique can be implemented with moderate computational complexity and only a small amount of feedback is required from the receive nodes.

Another more recent interference management approach is to use interference alignment (IA). It is shown in [5] that by using IA, the capacity of a $K$-user single-input and singleoutput interference channel with frequency selective or timevarying channel coefficients is

$$
C(S N R)=\frac{K}{2} \log _{2}(S N R)+o\left(\log _{2}(S N R)\right)
$$

which approaches the Shannon capacity of interference networks at high SNR. The main idea of IA is to align the interference into a reduced dimensional subspace by linear precoding so that simultaneous communications among many users over a small signal space can be achieved while keeping the desired signal separable from the interference [6].

Since both opportunistic transmission and IA require feedback, and this feedback is typically based on noisy estimates and is often coarsely quantized, it is of interest to understand how these systems perform in the presence of channel estimation or quantization errors. In [7], an opportunistic transmission scheduling policy is shown to be robust to estimation errors from both stochastic approximation algorithm and imperfect measurement of channel conditions. Another study [8] considers a broadcast channel with estimation errors where the transmit node sends to the user with the highest estimated SNR but backs off on the transmit rate based on the variance of the estimation error. The performance of such a scheme relies heavily on the duration of training period. For IA, the achievable sum mutual information, as well as upper and lower bounds are derived in [9] for MIMO interference channels with imperfect channel knowledge. In [10], the impact of imperfect channel state information (CSI) and channel correlation is quantified for MIMO IA. In contrast to this paper which focuses on SISO interference channels with closed-form IA precoding solutions, the focus of these studies is on MIMO interference channels and iterative algorithms for finding IA precoders.

In this paper, the performance of opportunistic transmission and IA with channel estimation errors is compared for a 3-user SISO interference system. A simple opportunistic transmission strategy is employed where only the transmitreceive pair with the largest channel magnitude estimate can communicate at each instant in time. For IA, a closed-form 
solution of a suboptimal subspace design [11] is used which avoids the initialization considerations inherent in iterative IA algorithms [12], [13]. Our analysis and simulation results for independent identically fading channels show that IA can achieve higher average sum rate only at high SNR and with accurate channel information whereas opportunistic transmission can provide better performance at low SNR and/or with relatively bad channel estimates.

The following notations are used in this paper: the upper case letters denote matrices, lower cases letters denote scalars, boldface letters denote vectors and $(.)^{\dagger}$ refers to the conjugate transpose of (.). Table I also lists the abbreviations used in this paper.

TABLE I

ABBREVIATIONS USED IN THIS PAPER.

\begin{tabular}{l||l} 
Abbreviation & Meaning \\
\hline CSI & Channel state information \\
IA & Interference alignment \\
CJ & $\begin{array}{l}\text { Joint original Cadambe-Jafar SISO IA solution } \\
\text { from [5] with SHV orthonormalization from [14] } \\
\text { and with perfect CSI }\end{array}$ \\
& $\begin{array}{l}\text { Joint improved SISO IA suboptimal subspace } \\
\text { optimization solution from [11] with SHV or- } \\
\text { thonormalization from [14] and with perfect CSI }\end{array}$ \\
OpT & $\begin{array}{l}\text { Opportunistic transmission [4] with perfect CSI } \\
\text { Random time-division channel access scheme } \\
\text { average sum rates with channel estimation error } \\
\text { of the corresponding scheme }\end{array}$ \\
RND &
\end{tabular}

\section{SYSTEM MODEL}

We assume a system with $K=3$ single-antenna transmitters and 3 single-antenna receivers where each transmitter wishes to send messages only to its associated receiver as shown in Figure 1. Let $h_{k j}(t) \stackrel{\text { i.i.d. }}{\sim} \mathcal{C N}(0, I)$ denote the channel from transmitter $j$ to receiver $k$ at time $t$ where $j, k \in\{1,2,3\}$. We assume that all channels are additive and that there is no intersymbol interference. A coarse level synchronization is assumed among the transmitters and receivers so that symbols arrive at the same time at the receivers.

\section{OpPORTUNISTIC TRANSMISSION}

Opportunistic transmission is a simple strategy that can improve average rate through by selecting the best available channel for transmission and only using this channel while the other transmitters remain silent. In the context of the system model in Section II, this means that only the transmitter with the maximum $\left|h_{k k}(t)\right|$ transmits at time $t$. Unlike interference alignment, as discussed in Section IV, opportunistic transmission only requires feedback of three channel states. We consider a scenario here with only spatial opportunism and no temporal opportunism. The transmitter with the best channel to its receiver transmits with fixed power $p$ in that timeslot.

For all $j, k \in\{1,2,3\}$, we have

$$
\begin{aligned}
f_{\left|h_{k j}(t)\right|}(x) & =\frac{x}{\sigma^{2}} e^{\frac{-x^{2}}{2 \sigma^{2}}}, \text { and } \\
F_{\left|h_{k j}(t)\right|}(x) & =1-e^{-\frac{x^{2}}{2 \sigma^{2}}}
\end{aligned}
$$

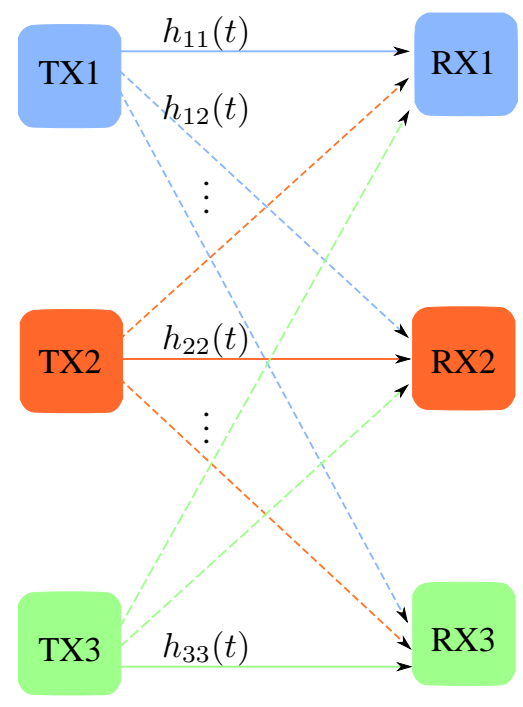

Fig. 1. A 3-user interference channel.

where $\sigma^{2}=0.5$. Letting

$$
\lambda(t)=\max \left(\left|h_{11}(t)\right|,\left|h_{22}(t)\right|,\left|h_{33}(t)\right|\right),
$$

we can write the distribution of the best channel as [15]

$$
f_{\lambda(t)}(x)=\frac{3 x}{\sigma^{2}} e^{-\frac{x^{2}}{2 \sigma^{2}}}\left(1-e^{-\frac{-x^{2}}{2 \sigma^{2}}}\right)^{2} .
$$

Under our fixed transmit power assumption, the average sum rate of the opportunistic transmission with perfect CSI can then be written as

$$
\begin{aligned}
R_{\text {ave-OT }} & =\mathrm{E}\left[\log _{2}\left(1+K p \lambda^{2}(t)\right)\right] \\
& =\int_{0}^{+\infty} f_{\lambda(t)}(x) \log _{2}\left(1+K p x^{2}\right) d x .
\end{aligned}
$$

Note that $R_{\text {ave-OT }}$ corresponds to the average sum rate of opportunistic transmission with perfect CSI.

When CSI is imperfect, there is a non-zero probability that the transmitter with the best channel is not selected for transmission. Let $\hat{h}_{k k}(t)$ denote the channel estimates from transmitter $k$ to receiver $k$ where $k \in\{1,2,3\}$. Define

$$
\hat{k}=\arg \max _{k \in\{1,2,3\}}\left|\hat{h}_{k k}(t)\right|
$$

and $\hat{\lambda}(t)=\left|h_{\hat{k} \hat{k}}(t)\right|$. Note that $\hat{\lambda}(t) \leq \lambda(t)$ for all $t$. Then the average sum rate of the opportunistic transmission with imperfect CSI can be written as

$$
\hat{R}_{\text {ave-OT-est }}=\mathrm{E}\left[\log _{2}\left(1+K p \hat{\lambda}^{2}(t)\right)\right] .
$$

\section{Three-user SiSO Interference Alignment}

This section describes the symbol extended channel model used for a 3-user SISO IA scheme, the associated performance metrics, and a closed-form solution for SISO IA precoding vectors. It is worth mentioning here that SISO IA requires feedback of all nine of the channels to all of the transmitters to allow computation of the precoding vectors. Channel 
estimation error can result in interference leakage. We also point out that, unlike opportunistic transmission where the transmit power was fixed in each timeslot, the IA scheme described below is based on an average power constraint for each symbol-extended block transmission.

\section{A. Symbol extended channel model}

To provide the required dimensionality for aligning interference subspaces in the SISO IA context, it is necessary to consider a symbol extended channel model in which each transmitter sends a block of precoded symbols over the channel. Let $X_{j}$ denote the $N$-symbol extension of the transmitted symbol $\boldsymbol{x}_{j}=\left[\begin{array}{lll}x_{j}(t+1) & \cdots & x_{j}\left(t+l_{j}\right)\end{array}\right]^{\top}$ from transmitter $j$ where $l_{j}$ represents the number of independent streams at the $j^{\text {th }}$ transmitter. It has been shown in [5] that $\left(l_{1}, l_{2}, l_{3}\right)=$ $(n+1, n, n)$ is achievable on the $N$-symbol extended channel when $N=2 n+1$. The elements of $\boldsymbol{x}_{j}$ are assumed to be i.i.d zero mean complex circularly symmetric Gaussian with variance $p$, i.e., $\boldsymbol{x}_{j} \sim \mathcal{C N}\left(0, p I_{l_{j}}\right)$. Hence, $X_{j}$ can be written as

$$
X_{j}=V_{j} \boldsymbol{x}_{j}=\left[\begin{array}{llll}
\boldsymbol{v}_{j}^{[1]} & \boldsymbol{v}_{j}^{[2]} & \cdots & \boldsymbol{v}_{j}^{\left[l_{j}\right]}
\end{array}\right] \boldsymbol{x}_{j}
$$

where $V_{j}$ is a $N \times l_{j}$ precoding matrix and $\boldsymbol{v}_{j}^{[i]}$ represents the $i^{\text {th }}$ column of $V_{j}, i \in\left\{1,2, \cdots, l_{j}\right\}$. Let $V_{j}^{\dagger} V_{j}=\frac{N}{l_{j}} I_{l_{j}}$. Since each transmitter access the channel $N$ times in a block transmission, the transmit power at transmitter $j$ is constrained such that $\mathrm{E}\left[\left\|X_{j}\right\|^{2}\right]=N p$.

Since the symbols from each transmitter are transmitted over $N$ time slots as a "supersymbol", the extended channel $H_{k j}$ (note that $H_{k j}$ is not MIMO channel) is defined as

$$
H_{k j}:=\left[\begin{array}{cccc}
h_{k j}(t+1) & 0 & \cdots & 0 \\
0 & h_{k j}(t+2) & \cdots & 0 \\
0 & 0 & \cdots & h_{k j}(t+N)
\end{array}\right]
$$

where $h_{k j}$ is a scalar at each channel use and $h_{k j}(t)$ and $h_{k j}(s)$ are independent for all $t \neq s$. The received signal vector at receiver $k$ is then

$$
\begin{aligned}
\boldsymbol{y}_{k} & :=H_{k k} X_{k}+\sum_{j \neq k}^{K} H_{k j} X_{j}+\boldsymbol{w}_{k} \\
& =H_{k k} V_{k} \boldsymbol{x}_{k}+\sum_{j \neq k}^{K} H_{k j} V_{j} \boldsymbol{x}_{j}+\boldsymbol{w}_{k}
\end{aligned}
$$

where $\boldsymbol{y}_{k}$ is the $N \times 1$ received signal vector and $\boldsymbol{w}_{k}$ is the additive white Gaussian noise at receiver $k$ distributed as $\boldsymbol{w}_{k} \sim \mathcal{C N}\left(0, I_{N}\right)$ at receiver $k$.

At the receiver side, we assume zero-forcing decoders. Let $U_{k}$ be an $N \times l_{k}$ matrix whose columns are orthogonal to the interference signal subspace at the $k^{t h}$ receiver. The filtered received signals can then be written as

$$
\begin{aligned}
\boldsymbol{z}_{k} & =U_{k}^{\dagger} \boldsymbol{y}_{k} \\
& =U_{k}^{\dagger} H_{k k} V_{k} \boldsymbol{x}_{k}+\sum_{j \neq k}^{K} U_{k}^{\dagger} H_{k j} V_{j} \boldsymbol{x}_{j}+U_{k}^{\dagger} \boldsymbol{w}_{k} .
\end{aligned}
$$

\section{B. Performance metrics}

If perfect knowledge of CSI is assumed at the transmitter and receiver, the individual sum rate at user $k$ derived with receivers deploying zero-forcing decoders can be written as

$$
R_{k}=\log _{2}\left|I_{l_{k}}+p \bar{H}_{k k} \bar{H}_{k k}^{\dagger}\left(\sum_{k \neq j} p \bar{H}_{k j} \bar{H}_{k j}^{\dagger}+I_{l_{k}}\right)^{-1}\right|
$$

where $\bar{H}_{k j}=U_{k}^{\dagger} H_{k j} V_{j}, \forall(k, j)$. The average sum rate with perfect CSI is then

$$
R_{\text {ave-IA }}=\frac{1}{N} \mathrm{E}\left[\sum_{k=1}^{K} R_{k}\right] .
$$

When CSI is imperfect, we can denote the channel estimate as $\hat{H}_{k j}$. Then the precoding and decoding matrices $\hat{V}_{j}$ and $\hat{U}_{k}$, respectively, are computed based on the estimated CSI rather than the actual CSI. Hence the resulting individual sum rate will be

$$
\hat{R}_{k}=\log _{2}\left|I_{l_{k}}+p \hat{\bar{H}}_{k k} \hat{\bar{H}}_{k k}^{\dagger}\left(\sum_{k \neq j} p \hat{\bar{H}}_{k j} \hat{\bar{H}}_{k j}^{\dagger}+I_{l_{k}}\right)^{-1}\right|
$$

where $\hat{\bar{H}}_{k j}=\hat{U}_{k}^{\dagger} H_{k j} \hat{V}_{j}, \forall(k, j)$. The corresponding average sum rate with imperfect CSI is then

$$
\hat{R}_{\text {ave-IA-est }}=\frac{1}{N} \mathrm{E}\left[\sum_{k=1}^{K} \hat{R}_{k}\right] .
$$

\section{Closed-form solutions for SISO IA}

Many algorithms for computing optimal IA precoding matrices are iterative and, since convergence depends on the initialization, this makes them unattractive when it comes to studying the performance of IA with imperfect CSI. In this section, a handful of non-iterative IA algorithms with closedform solutions will be discussed and used as a basis for our comparison with opportunistic transmission in the sequel.

SISO interference alignment with $l_{k}$ streams from transmitter $k$ requires

$$
\begin{aligned}
U_{k}^{\dagger} H_{k j} V_{j} & =0 \quad \text { for } j \neq k \\
\operatorname{rank}\left(U_{k}^{\dagger} H_{k k} V_{k}\right) & =l_{k} .
\end{aligned}
$$

The first closed-form solution for the precoding vectors in a 3-user SISO interference channel was given in [5] in which the precoding vectors are defined as

$$
\left.\begin{array}{l}
H_{12} V_{2}=H_{13} V_{3} \\
H_{23} V_{3} \prec H_{21} V_{1} \\
H_{32} V_{2} \prec H_{31} V_{1}
\end{array}\right\} \Rightarrow \begin{aligned}
& V_{1}=A \\
& V_{2}=\left(H_{32}\right)^{-1} H_{31} C \\
& V_{3}=\left(H_{23}\right)^{-1} H_{21} B
\end{aligned}
$$

where

$$
\begin{aligned}
T: & =H_{12}\left(H_{21}\right)^{-1} H_{23}\left(H_{32}\right)^{-1} H_{31}\left(H_{13}\right)^{-1} \\
A: & =\left[\begin{array}{lllll}
\boldsymbol{\omega} & T \boldsymbol{\omega} & \cdots & T^{n} \boldsymbol{\omega}
\end{array}\right]_{N \times(n+1)} \\
B: & =\left[\begin{array}{lllll}
T \boldsymbol{\omega} & T^{2} \boldsymbol{\omega} & \cdots & T^{n} \boldsymbol{\omega}
\end{array}\right]_{N \times n} \\
C: & =\left[\begin{array}{llll}
\boldsymbol{\omega} & T \boldsymbol{\omega} & \cdots & T^{n-1} \boldsymbol{\omega}
\end{array}\right]_{N \times n}
\end{aligned}
$$


In [5], $\boldsymbol{\omega}=\left[\begin{array}{llll}1 & 1 & \cdots & 1\end{array}\right]^{\top}$.

Based on this scheme, it is showed in [16] that a global optimal solution $\tilde{\boldsymbol{\omega}}^{*}$ exists which maximizes the sum rate while preserving the achievable degrees of freedom. An alternative suboptimal improved subspace design is proposed in [11] where the suboptimal precoding vector is

$$
\begin{aligned}
V_{k}(\boldsymbol{\omega}) & =W(\boldsymbol{\omega}) \Gamma_{k} \\
\tilde{\omega}_{i} & =3\left(\sum_{k} \gamma_{k i} \gamma_{k i}^{\dagger}\right)^{-1}
\end{aligned}
$$

where $\tilde{W}:=W^{\dagger} W, \tilde{\omega}_{i}=\left|\omega_{i}\right|^{2}$ which is the $i$ th element of the $N \times N$ diagonal matrix $\tilde{W}$ and $\gamma_{k i}$ is the $i$ th row vector of matrix $\Gamma_{k}$ (which is defined in [16]). Both CJ and KT schemes can be further improved by SHV orthonormalization [14].

Figure 2 shows the average sum rate of the combined $\mathrm{CJ} / \mathrm{SHV}$ and KT/SHV scheme, denoted as CJ and KT in the plot respectively, for a 3-user SISO IA with $N=3$ at various SNRs. The degrees of freedom are defined as

$$
D_{n}=\frac{\sum_{k=1}^{K} l_{k}}{N}=\frac{3 n+1}{2 n+1} .
$$

The ideal sum rate $D_{n} \log _{2}(\mathrm{SNR})$ is also plotted as reference where $D_{1}=\frac{4}{3}$ in this case.

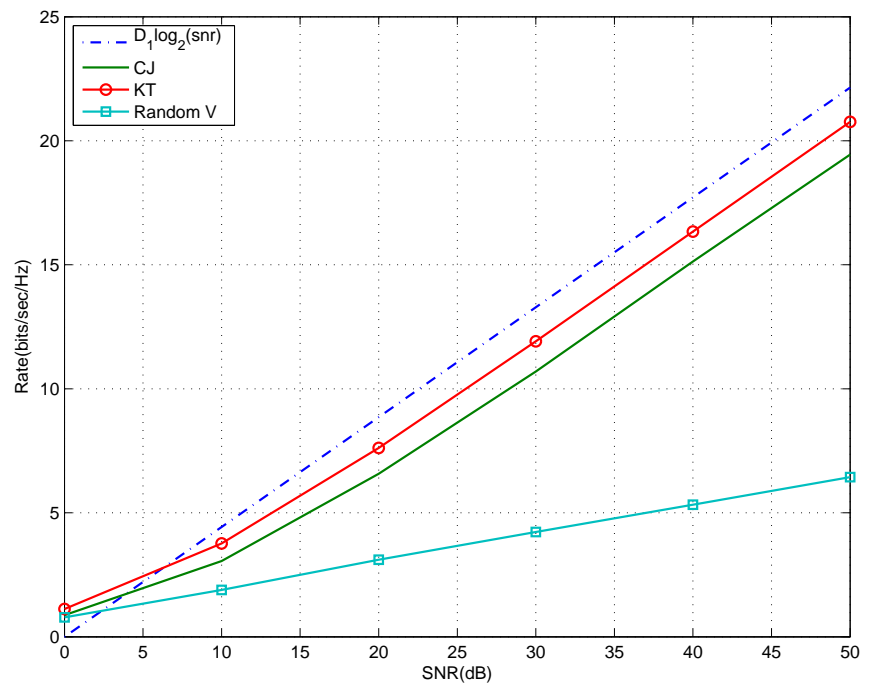

Fig. 2. Average sum rate of SISO IA techniques for a 3-user interference channel $N=3$ symbol extensions.

\section{NUMERICAL RESULTS}

In this section, we compare the average sum rates obtained by the opportunistic transmission and SISO IA schemes with ZF decoder.

In the first example, a 3-user SISO interference channel is assumed in the system where each transmitter has $N=3$ symbol extensions. Channel coefficients are drawn temporarily and spatially i.i.d. from a complex Gaussian distribution, i.e., $h_{k j}(t) \stackrel{\text { i.i.d. }}{\sim} \mathcal{C N}\left(0, I_{N}\right)$. Independent circularly symmetric complex channel estimation errors denoted $w_{k j}(t) \stackrel{\text { i.i.d. }}{\sim}$. $\mathcal{C N}\left(0, \sigma_{W}^{2}\right)$ as are assumed. 1000 channel realizations are generated and 1000 noise realizations are generated for each $\sigma_{W}^{2}$ value.

As shown in Figure 3 for $N=3$, the KT SISO IA scheme achieves the highest average sum rate as $16.03 \mathrm{bits} / \mathrm{sec} / \mathrm{Hz}$ which has a gain of $0.57 \mathrm{bits} / \mathrm{sec} / \mathrm{Hz}$ over the OT and a gain of $1.35 \mathrm{bits} / \mathrm{sec} / \mathrm{Hz}$ over CJ respectively at $40 \mathrm{~dB}$ SNR. When channel estimation error is considered, the average data rate performance decreases as expected. Compared with KTest, OT-est is about $1 \mathrm{bits} / \mathrm{sec} / \mathrm{Hz}$ worse when $\sigma_{W}^{2} \leq 10^{-4}$. However, the OT-est outperforms KT-est when the channel estimates get worse. When $\sigma^{2}>10^{-4}$, OT-est starts to outperform KT-est and is also robust to the bad channel estimates. As $\sigma_{W}^{2}$ increases, OT-est converges to the RND scheme which picks a random user to transmit at full power. The two SISO IA schemes shown in Figure 3 perform worse than RND when $\sigma_{W}^{2}>0.1$.

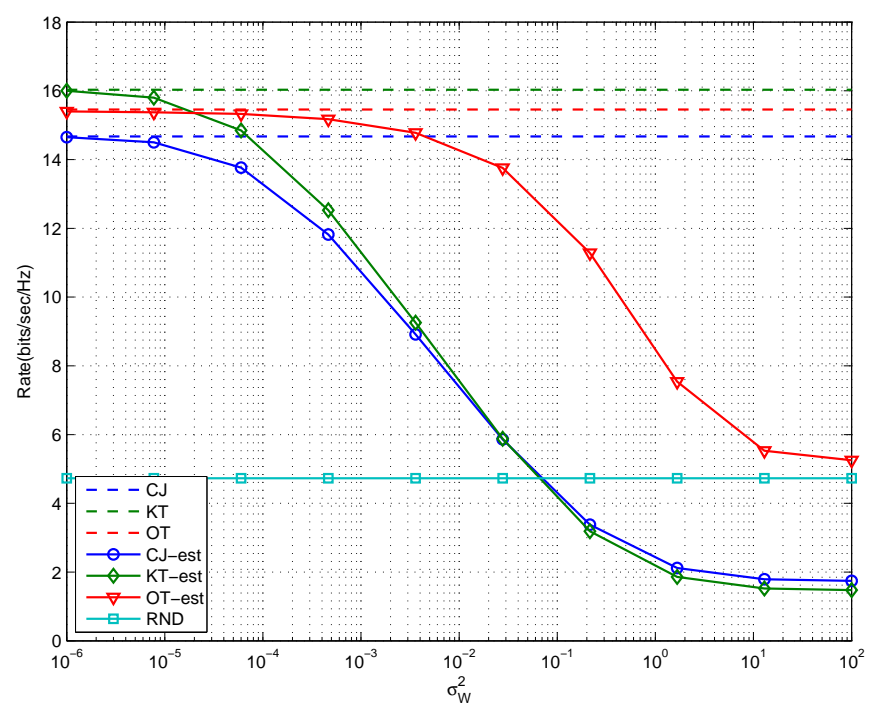

Fig. 3. Average sum rate versus channel estimation error variance for 3-user opportunistic transmission and SISO IA with $N=3$ symbol extensions at $40 \mathrm{~dB}$ SNR.

Figure 4 shows the same simulation as Figure 3 except the SNR is now set to $15 \mathrm{~dB}$. Here we see opportunistic transmission performing better than both SISO IA schemes. The average sum rate for KT is almost $1.76 \mathrm{bits} / \mathrm{sec} / \mathrm{Hz}$ worse than the OT. This gain maintains for the imperfect CSI cases, i.e., OT-est always at least $1 \mathrm{bits} / \mathrm{sec} / \mathrm{Hz}$ better than KT-est. Again, OT-est converges to RND as $\sigma_{W}^{2}$ increases.

Figure 5 shows the average sum rate performance for the 3-user SISO IA with $N=5$ symbol extensions at $40 \mathrm{~dB}$. Compared with Figure 3, KT is about $0.45 \mathrm{bits} / \mathrm{sec} / \mathrm{Hz}$ better than the $N=3$ case and OT-est starts to outperform KTest around $\sigma_{W}^{2}=10^{-4}$. The CJ, however, is $1.47 \mathrm{bits} / \mathrm{sec} / \mathrm{Hz}$ worse than that in the $N=3$ case. Hence increasing the number of symbol extensions $N$ does not always improve the average sum rate performance. Even for the KT scheme, the 


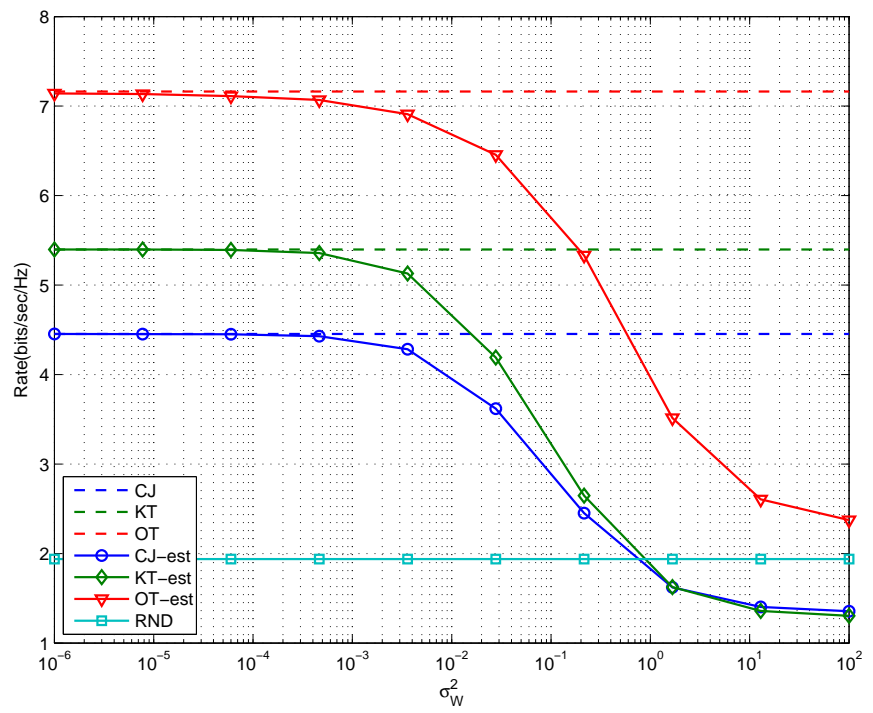

Fig. 4. Average sum rate versus channel estimation error variance for 3-user opportunistic transmission and SISO IA with $N=3$ symbol extensions at $15 \mathrm{~dB}$ SNR.

average sum rate will drop when $N$ is greater than the number of sources of interference, i.e. $K(K-1)$.

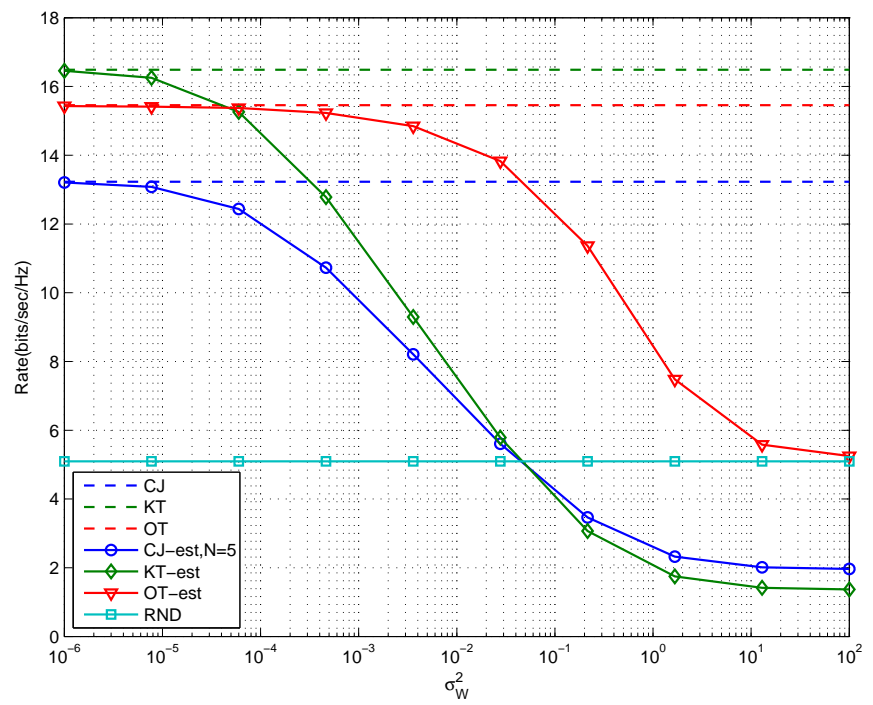

Fig. 5. Average sum rate versus channel estimation error variance for 3-user opportunistic transmission and SISO IA with $N=5$ symbol extensions at $40 \mathrm{~dB}$ SNR.

In the remaining results, we directly compare the sum rate of different schemes by varying SNR from 0 to $40 \mathrm{~dB}$ and channel estimation error variance $\sigma_{W}^{2} \in\left[10^{-6}, 1\right] .1000$ channel/noise realizations are performed for each (SNR, $\sigma_{W}^{2}$ ) pair. For $N=3$ symbol extensions, the difference of the average sum rate between OT and CJ and OT and KT are compared in Figure 6 and 7, respectively. A positive contour indicates that the OT outperforms IA techniques in terms of average sum rate, whereas a negative contour indicates that IA is better. Between the two IA schemes, KT achieves slightly larger performance advantage area over OT in the regime of good channel estimates and high SNR, i.e., $\sigma_{W}^{2}<10^{-4}$ and $\mathrm{SNR}>30 \mathrm{~dB}$. With low SNR and/or poor channel estimates, i.e., $\mathrm{SNR}<30 \mathrm{~dB}$ and/or $\sigma_{W}^{2}>10^{-4}$, OT has the best sum rate performance amongst all schemes considered here. Figure 8 shows the difference of the average sum rate between OT and KT for the case with $N=5$ symbol extensions. As can be seen, KT has slightly better performance than the $N=3$ case at high SNR and in the low $\sigma_{W}^{2}$ regime. Otherwise, the results are similar to those seen in the $N=3$ case. When the SNR is low or the channel estimates are bad, opportunistic transmission achieves better sum rate performance than either SISO IA scheme.

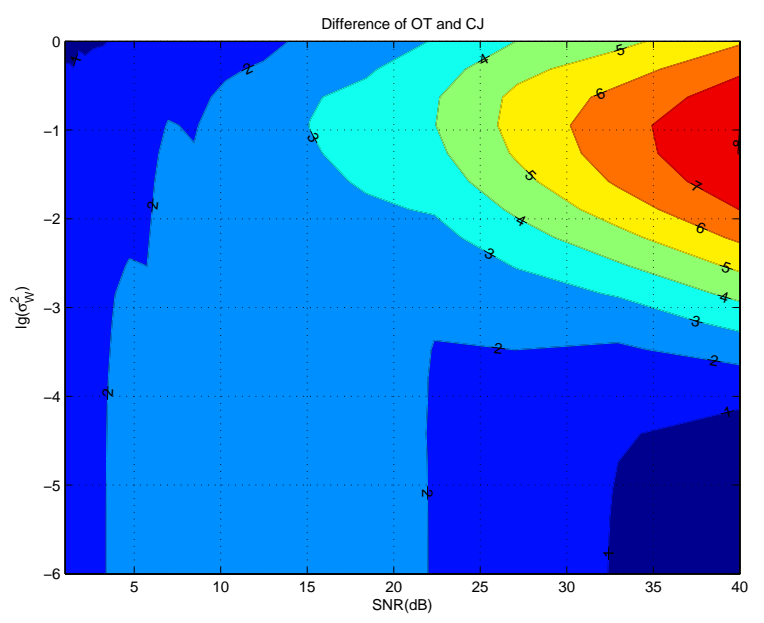

Fig. 6. The difference of the average sum rate between OT and CJ with $N=3$ symbol extensions.

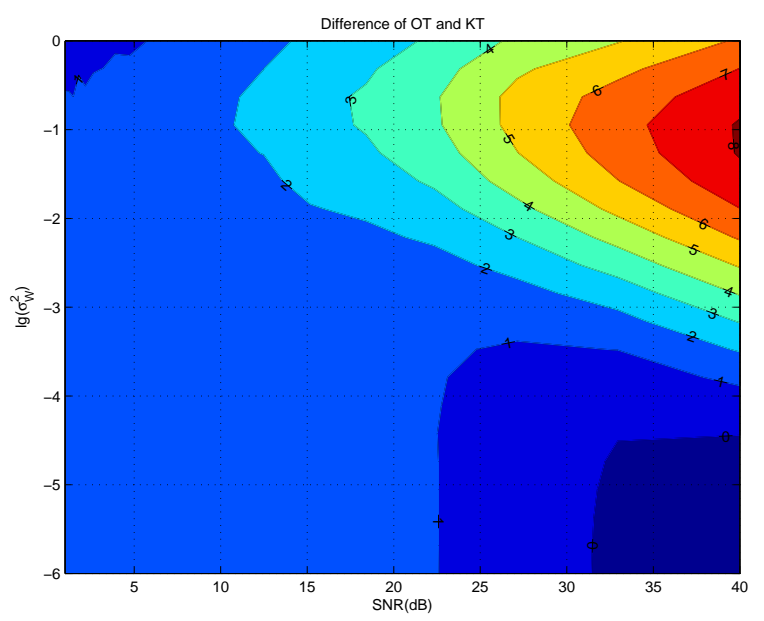

Fig. 7. The difference of the average sum rate between OT and KT with $N=3$ symbol extensions.

\section{CONCLUSION}

In this paper, we have compared opportunistic transmission with a low complexity subspace suboptimal IA technique based the 3-user SISO IA scheme proposed in [5]. Simulation 


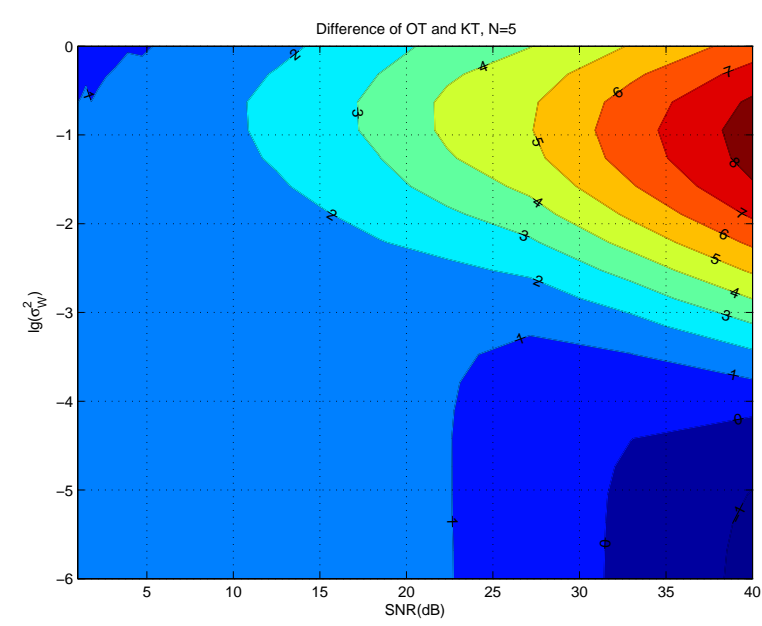

Fig. 8. The difference of the average sum rate between OT and KT with $N=5$ symbol extensions.

results show that opportunistic transmission outperforms SISO IA in low SNR conditions and/or when channel estimates are poor. SISO IA based on [5] or [11] with SHV orthonormalization tends to perform better at high SNR with good channel estimates.

\section{REFERENCES}

[1] G. Boudreau, J. Panicker, N. Guo, R. Chang, N. Wang, and S. Vrzic, "Interference coordination and cancellation for $4 \mathrm{~g}$ networks," Соттиnications Magazine, IEEE, vol. 47, no. 4, pp. 74 -81, April 2009.

[2] J. Andrews, "Interference cancellation for cellular systems: a contemporary overview," Wireless Communications, IEEE, vol. 12, no. 2, pp. 19 - 29, April 2005.

[3] Q. Zhang, Q. Chen, F. Yang, X. Shen, and Z. Niu, "Cooperative and opportunistic transmission for wireless ad hoc networks," Network, IEEE, vol. 21, no. 1, pp. 14 -20, Janurary 2007.

[4] L. Dong, T. Li, and Y.-F. Huang, "Opportunistic transmission scheduling for multiuser mimo systems," in Acoustics, Speech, and Signal Processing, 2003. Proceedings. (ICASSP '03). 2003 IEEE International Conference on, vol. 5, April 2003, pp. V - 65-8 vol.5.

[5] V. Cadambe and S. Jafar, "Interference alignment and spatial degrees of freedom for the k user interference channel," in Communications, 2008. ICC '08. IEEE International Conference on, May 2008, pp. 971 -975.

[6] S. Jafar, Interference Alignment A New Look at Signal Dimensions in a Communication Network. Now Publishers Inc, 2011.

[7] X. Liu, E. Chong, and N. Shroff, "Opportunistic transmission scheduling with resource-sharing constraints in wireless networks," Selected Areas in Communications, IEEE Journal on, vol. 19, no. 10, pp. 2053 -2064, October 2001.

[8] A. Vakili, M. Sharif, and B. Hassibi, "The effect of channel estimation error on the throughput of broadcast channels," in Acoustics, Speech and Signal Processing, 2006. ICASSP 2006 Proceedings. 2006 IEEE International Conference on, vol. 4, May 2006, p. IV.

[9] R. Tresch and M. Guillaud, "Cellular interference alignment with imperfect channel knowledge," in Communications Workshops, 2009. ICC Workshops 2009. IEEE International Conference on, June 2009, pp. $1-5$.

[10] B. Nosrat-Makouei, J. Andrews, and R. Heath, "Mimo interference alignment over correlated channels with imperfect csi," Signal Processing, IEEE Transactions on, vol. 59, no. 6, pp. 2783 -2794, June 2011.

[11] D. Kim and M. Torlak, "Interference alignment via improved subspace conditioning," in Global Telecommunications Conference (GLOBECOM 2010), 2010 IEEE, December 2010, pp. 1 -5.

[12] S. Peters and R. Heath, "Interference alignment via alternating minimization," in Acoustics, Speech and Signal Processing, 2009. ICASSP 2009. IEEE International Conference on, April 2009, pp. 2445 -2448.
[13] _ "Cooperative algorithms for mimo interference channels," Vehicular Technology, IEEE Transactions on, vol. 60, no. 1, pp. 206 -218, Janurary 2011.

[14] S. Liu and Y. Du, "A general closed-form solution to achieve interference alignment along spatial domain," in Global Telecommunications Conference (GLOBECOM 2010), 2010 IEEE, December 2010, pp. 1 -5 .

[15] G. Casella and R. Berger, Statistical inference. Duxbury Press, 2001.

[16] D. Kim and M. Torlak, "Optimization of interference alignment beamforming vectors," Selected Areas in Communications, IEEE Journal on, vol. 28, no. 9, pp. 1425 -1434, December 2010. 